\title{
COMPARATIVE EFFECTIVENESS OF BREAST CONSERVING SURGERY VERSUS MASTECTOMY ON SURVIVAL IN BREAST CANCER PATIENTS THAT RECEIVED NEOADJUVANT CHEMOTHERAPY AND POST-OPERATIVE RADIATION THERAPY
}

Gustavo Nader Marta1,2, Allan A. Lima Pereira³, Leandro Jonata Oliveira33, José Roberto Filassi4 , Max S. Mano³

'Department of Radiation Oncology, Hospital Sírio-Libanês - São Paulo (SP), Brazil. 2Department of Radiology and Oncology, Radiation Oncology Unit, School of Medicine Universidade de São Paulo, Instituto do Câncer do Estado de São Paulo - São Paulo (SP), Brazil.

${ }^{3}$ Department of Clinical Oncology, Hospital Sírio-Libanês - São Paulo (SP), Brazil.

${ }^{4}$ Department of Obstetrics and Gynecology, Division of Breast Surgery, Instituto do Câncer do Estado de São Paulo, School of Medicine, Universidade de São Paulo - São Paulo (SP), Brazil.

Objectives: The aim of this study was to determine whether there were differences in survival outcomes of matched breast cancer patients undergoing breast-conserving surgery (BCS) versus mastectomy that received neoadjuvant chemotherapy (NAC). Methodology: A retrospective cohort of patients with stage I-III breast cancer treated between 2008 and 2014 at two institutions who had received NAC followed by surgery and post-operative radiation therapy was identified. KaplanMeier and multivariate Cox proportional hazards models were used to examine disease-free survival (DFS) and overall survival (OS) rates by surgery type. Results: Of 652 patients, 162 (24.9\%) underwent BCS and 490 (75.1\%) underwent mastectomy /adenomastectomy. Most of the patients ( $\mathrm{n}=589,91.1 \%$ ) had locally advanced disease (clinical stage IIB to IIIC) with a mean age of 50 years. In regards of surgery type, patients with stage III disease underwent more mastectomy them BCS ( $n=383,79.0 \%$ mastectomy versus $n=81,50.3 \%$ BCS; $p<0.001)$. The DFS and OS rates for all patients at 3 years were 81.5

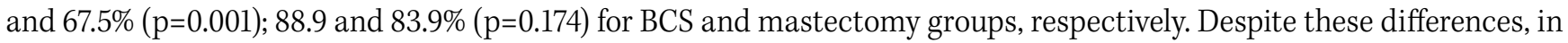
the multivariate analyzed adjusted by clinical stage and pathologic complete response, there were no statistical differences in DFS (mastectomy versus BCS HR 1.44; 95\%CI 0.95-2.17) and OS (mastectomy versus BCS HR 1.03; 95\%CI 0.60-1.75) concerning the surgery type. Conclusion: In breast cancer patients who underwent NAC and post-operative radiation therapy, BCS and mastectomy are effective with similar survival outcomes regardless of surgery type. 